\title{
Adiponectin Signaling Regulates Lipid Production in Human Sebocytes
}

Jeung-Hoon Lee ${ }^{1}$, Yu-Ra Jung ${ }^{1}$, Ji-Young Kim ${ }^{1}$, Young Lee ${ }^{1}$, Young-Joon Seo ${ }^{1}$, Chang-Deok Kim ${ }^{1}$, Seong-Jin Kim ${ }^{2}$, Myung $\operatorname{Im}^{1}$

${ }^{1}$ Department of Dermatology, College of Medicine, Chungnam National University, Daejeon, Korea

2 Department of Dermatology, Chonnam National University Medical School, Gwangju, Korea

(This study was supported by a grant of the Korea Healthcare technology R\&D Project, Ministry of Health \& Welfare, Republic of Korea, Grant number HI14C1379)

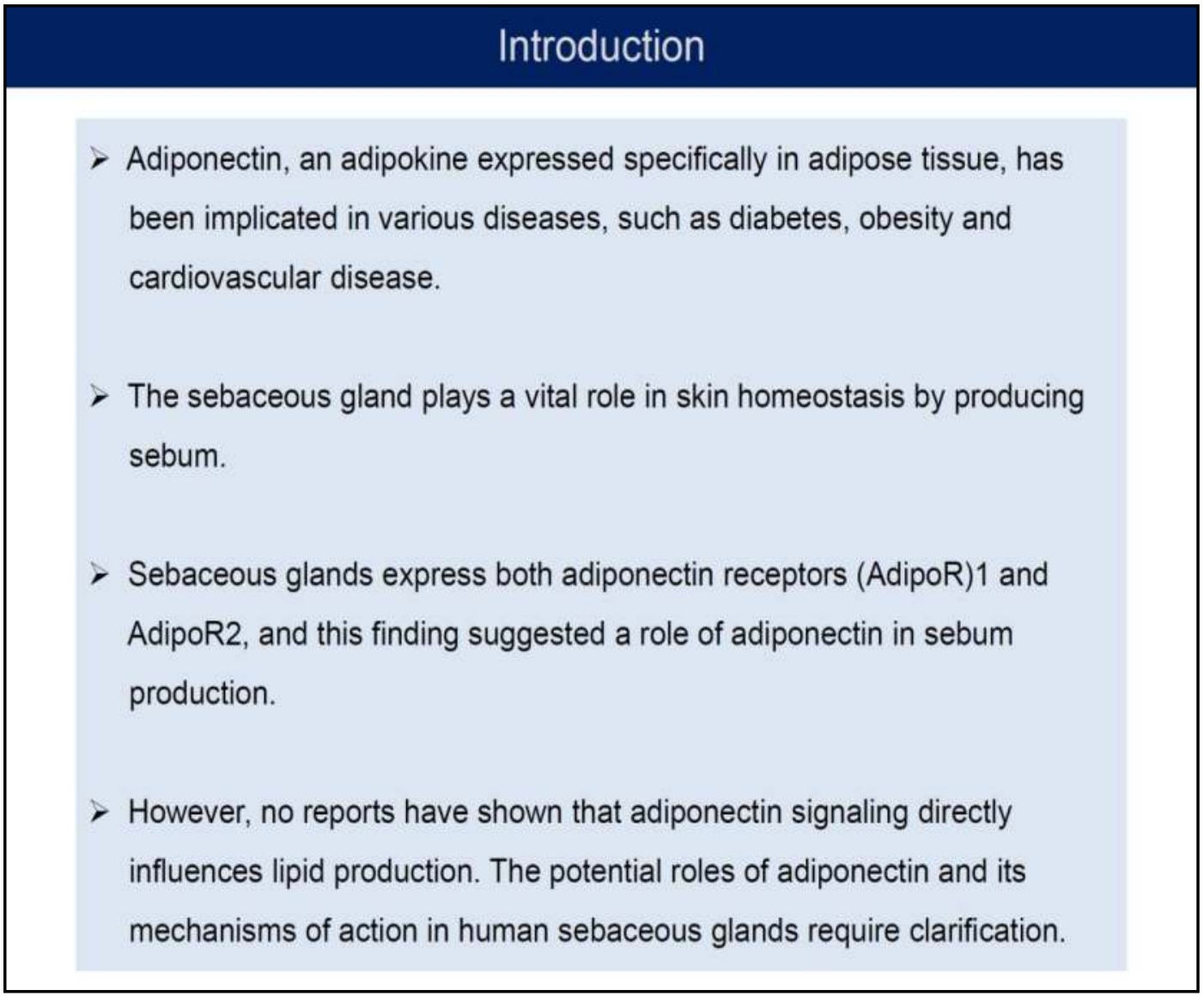

Expression of adiponectin receptors in human sebaceous glands and sebocytes
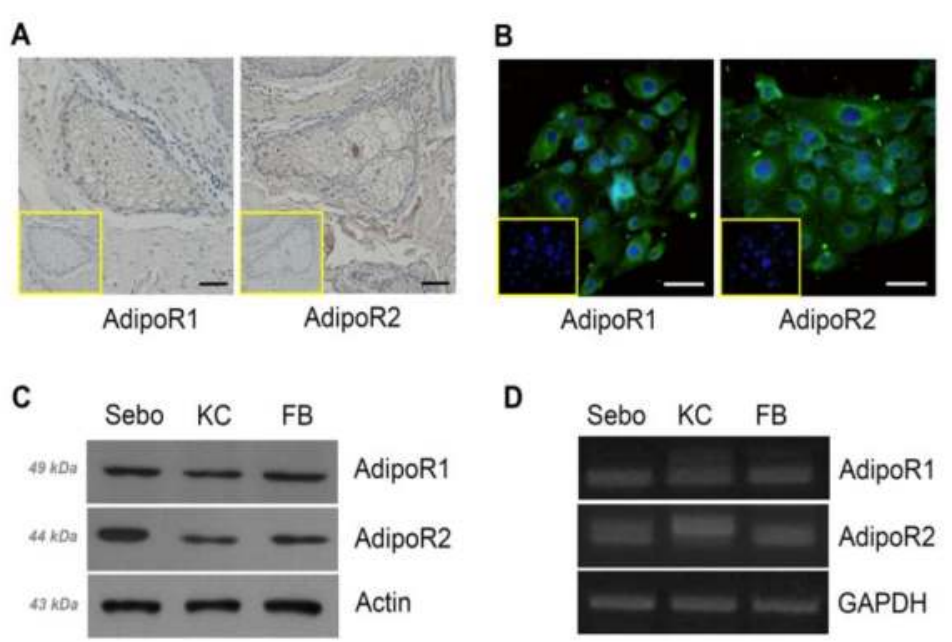

(A) Human sebaceous glands were assayed for adiponectin receptor (AdipoR)1 and Adipo R2 by immunohistochemistry. Inset,
isotype isotype control. (B) Immunofiuorescence labeling of AdipoR1 and AdipoR2 (green) in human sebocyles. Nuclel were
counterstained with DAPI (blue). Inset, isotype control. (C, D) Westem blotting and RT.PCR of sebocyte Iysates. Human keratinocytes and fibroblasts expressing AdipoR1 and AdipoR2 were used as positive controls. Scale bars $=20 \mu \mathrm{mm}$. Sebo
sebocytes; $\mathrm{KC}$, keratinocytes; $\mathrm{FB}$, fibroblasts.

Effects of adiponectin on lipid production in human sebocytes

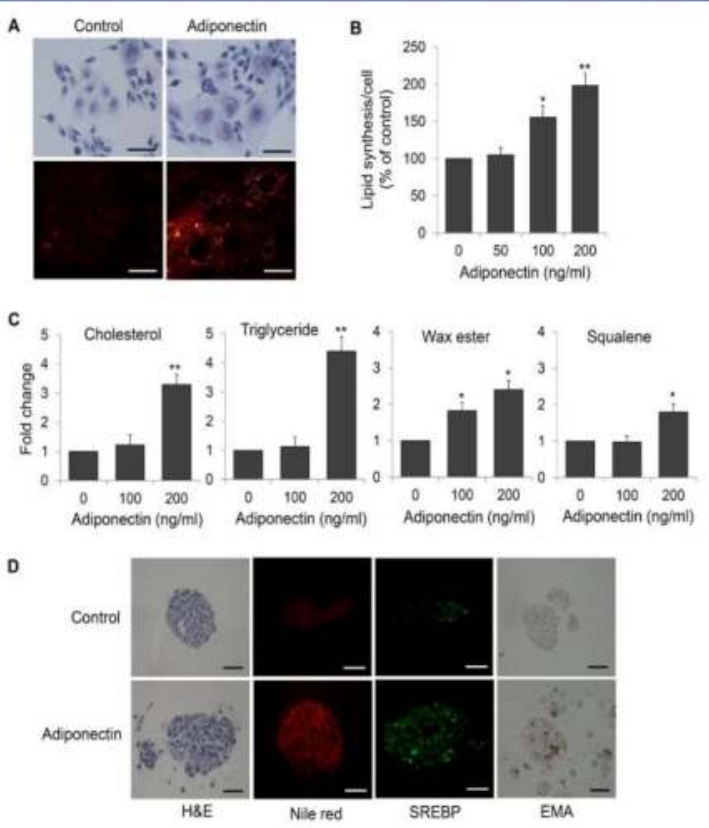

(A) Detection of intracellular lipids in sebocytes treated with adiponectin using microscopy after Oil Red $O$ and Nile red staining. (B)
Lipid levels in sebocyles treated with various doses of adiponectin. (C) Relative abundance of major lipid classes detetemined by thin-layer chromatography. (D) The effect of adiponectin in three-dimensional (3D) culture of human sebocyles. Organoid

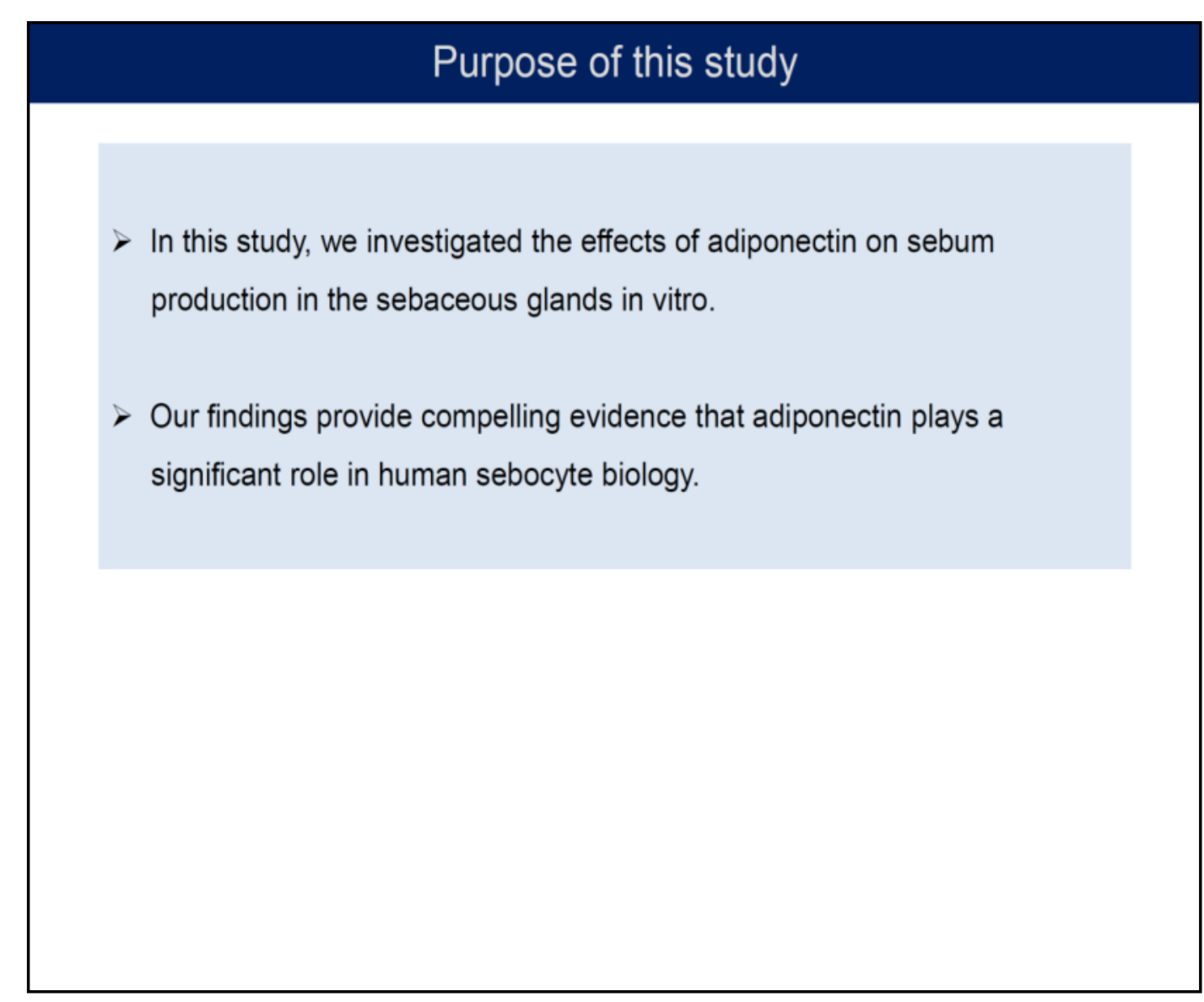

Effects of adiponectin on cell proliferation in human sebocytes
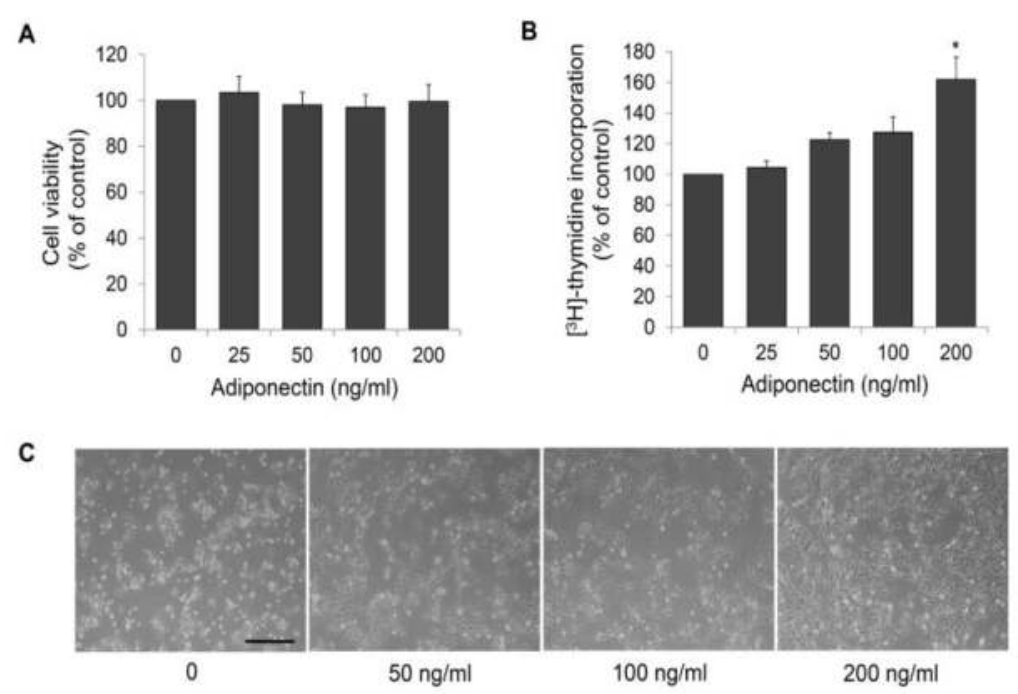

(A) The effects of adiponectin on sebocyte viability were examined by MTT assay. (B) Rate of $[$ PH]-thymidine incorporation into dependent effects of adiponectin on sebocyte proliferation were examined using microscopy from low cell seeding assays. Scale

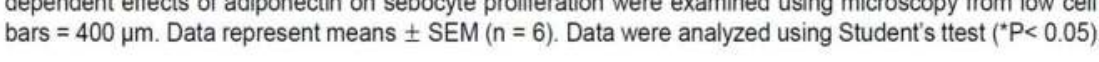

Induction of lipid synthesis by adiponectin in human sebocytes by activating APPL1-AMP activated protein kinase (AMPK) signaling

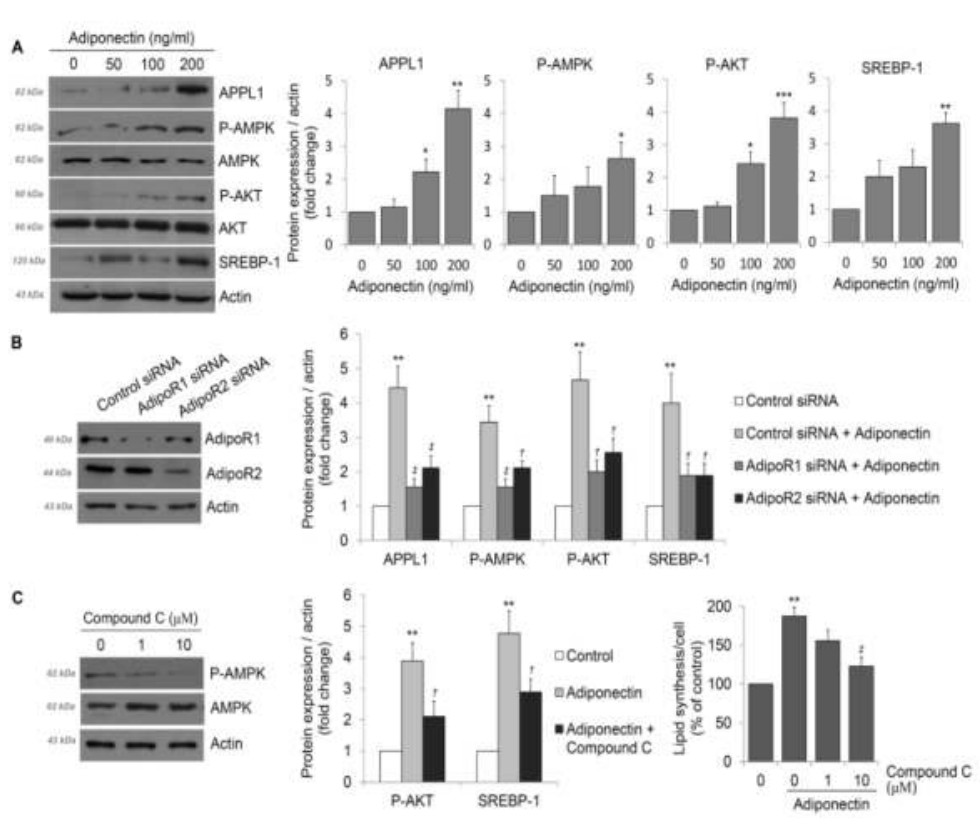

(A) After sebocyles were treated with adiponectin, whole cell lysates were prepared and analyzed by Western blotting. (B)

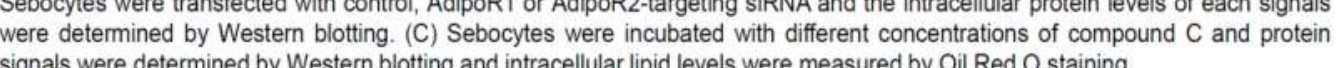

\section{Summary}

- We demonstrated that human sebaceous glands in vivo and sebocytes in vitro express adiponectin receptor and that adiponectin increased cell proliferation.

> Moreover, based on a lipogenesis study using Oil Red O, Nile red staining and thin layer chromatography, adiponectin strongly upregulated lipid production in sebocytes.

> In three-dimensional culture of sebocytes, lipid synthesis was markedly enhanced in sebocytes treated with adiponectin.

> This study suggested that adiponectin plays a significant role in human sebaceous gland biology.

> Adiponectin signaling is a promising target in the clinical management of barrier disorders in which sebum production is decreased, such as in atopic dermatitis and aged skin 